\title{
Evaluation of amplification refractory mutation system (ARMS) technique for quick and accurate prenatal gene diagnosis of $\mathrm{CHM}$ variant in choroideremia
}

This article was published in the following Dove Press journal:

The Application of Clinical Genetics

\author{
Lisha Yang' \\ Iqra ljaz' \\ Jingliang Cheng ${ }^{1,2}$ \\ Chunli Wei ${ }^{1,3}$ \\ Xiaojun Tan ${ }^{4}$ \\ Md Asaduzzaman Khan' \\ Xiaodong $\mathrm{Fu}^{5}$ \\ Junjiang $\mathrm{Fu}^{1-3}$
}

'Key Laboratory of Epigenetics and Oncology, the Research Center for Precision Medicine, Southwest Medical University, Luzhou, ${ }^{2}$ Department of Pathology, Hunan Normal University College of Medicine, Changsha, ${ }^{3}$ State Key Laboratory of Quality Research in Chinese Medicine, Macau University of Science and Technology, Macau (SAR), ${ }^{4}$ Reproductive and Genetic Center, the Central Hospital of Xiangtan City, Xiangtan, ${ }^{5}$ Department of Obstetrics and Gynecology, First Affiliated Hospital of Southwest Medical University, Luzhou, People's Republic of China
Correspondence: Junjiang Fu; Xiaodong Fu

Southwest Medical University, 3-319

Zhongshan Road, Luzhou City, China

646000

Tel +868303160283

Email fujunjiang@hotmail.com; dongerfu@I63.com

\begin{abstract}
Choroideremia is a rare X-linked recessive inherited disorder that causes chorioretinal dystrophy leading to visual impairment in its early stages which finally causes total blindness in the affected person. It is caused due to mutations in the CHM gene. In this study, we have recruited a pedigree with choroideremia and detected a nonsense variant (c.C799T:p.R267X) in $C H M$ of the proband (I:1). Different primer sets for amplification refractory mutation system (ARMS) were designed and PCR conditions were optimized. Then, we evaluated the sequence variant in the patient, carrier, and a fetus by using ARMS technique to identify if they inherited the pathogenic gene from parental generation; we used amniotic fluid DNA for the diagnosis of the gene in the fetus. The primer pairs, WT2 $+\mathrm{C}$ and $\mathrm{MT}+\mathrm{C}$, amplified high specific products in different DNAs which were verified by Sanger sequencing. Based on our results, ARMS technique is fast, accurate, and reliable prenatal gene diagnostic tool to assess $C H M$ variants. Taken together, our study indicates that ARMS technique can be used as a potential molecular tool in the diagnosis of prenatal mutation for choroideremia as well as other genetic diseases in undeveloped and developing countries, where there might be shortage of medical resources and supplies.

Keywords: choroideremia, $C H M$ gene, mutation, amplification refractory mutation system (ARMS), prenatal diagnosis, evaluation
\end{abstract}

\section{Introduction}

Choroideremia (CHM, OMIM: 303100) is a rare X-linked recessive inherited disorder that causes chorioretinal dystrophy with typical clinical features such as progressive degeneration of the choriocapillaris, retinal pigment epithelium (RPE), and photoreceptors. ${ }^{1}$ Its prevalence ranges from 1:50,000 to 1:100,000. ${ }^{2}$ Male patients develop night blindness in their childhood and gradually progress to loss of peripheral vision, and finally, some of them will reach complete peripheral blindness at their middle age with only tunnel vision left behind. Female carriers are usually asymptomatic, as to the heterozygous gene, some of them occasionally show mild symptoms as they reach middle age. ${ }^{3,4}$ Thus, with the progression of the disease, central vision is typically maintained in men as the fovea is spared.

$\mathrm{CHM}$ is caused by mutations in the $C H M$ gene, which is mapped on chromosome $\mathrm{Xq} 21.2-21.3$ in 186,382 bp. ${ }^{5}$ CHM mRNA comprises 15 exons, and it is expressed in many types of tissues including choroid, retinal photoreceptor, RPE, and lymphocyte tissues. ${ }^{6}$ Ran et al reported that there are more than 100 different pathogenic $C H M$ submit your manuscript | www.dovepress.com
The Application of Clinical Genetics 20 I8: I I I-8

(c) (i) (5) 2018 Yang et al. This work is published and licensed by Dove Medical Press Limited. The full terms of this license are available at https://www.dovepress.com/terms. (C) php and incorporate the Creative Commons Attribution - Non Commercial (unported, v3.0) License (http://creativecommons.org//icenses/by-nc/3.0/). By accessing the work you hereby accept the Terms. Non-commercial uses of the work are permitted without any further permission from Dove Medical Press Limited, provided the work is properly attributed. For permission for commercial use of this work, please see paragraphs 4.2 and 5 of our Terms (https://www.dovepress.com/terms.php). 
variants in the RetinoGenetics. ${ }^{7}$ These variants demonstrate deletions, translocations, missense mutations, nonsense mutations, and splice-site mutations ${ }^{6,8,9}$ and have been identified in the 15-exon gene. The CHM gene encodes for Rab escort protein-1 (REP1) which comprises 653 amino acids. ${ }^{10}$ REP1 plays a significant role in the intracellular vesicular trafficking process as well as in the post-translational isoprenyl modification of Rab proteins. ${ }^{11,12}$

Nowadays, varieties of molecular techniques have been invented to detect genetic mutations, for example, Sanger sequencing, but this technique cannot rapidly screen large numbers of mutations in samples. Fortunately, this problem can be solved by massive next-generation sequencing (NGS), ${ }^{13}$ but both Sanger sequencing and NGS are expensive. The amplification refractory mutation system (ARMS), also termed allele-specific PCR, is a very simple method for detecting any known mutations involving single base changes or small deletions, which is based on the use of sequencespecific PCR primers that allow amplification of test DNA only when the target allele is contained within the sample. ARMS technique demands only PCR amplification and gel electrophoresis of the amplicons.

In ARMS technique, one PCR comprises one allelespecific oligonucleotide primer at $5^{\prime}$-end and a common primer at $3^{\prime}$-end. If the presence of an amplified mutant is detected by agarose gel electrophoresis, it suggests that the target sequence contains the mutant allele. Similarly, if the result displays an absence of the amplified mutant, it indicates the presence of the normal DNA sequence on that specific point.
In the same way, a normal primer at $5^{\prime}$-end together with a common primer at 3'-end was used in another PCR. If normal amplified product is present, it reveals the existence of a natural DNA sequence, whereas if normal amplified product is absent, then it reveals the presence of a mutant allele. ${ }^{14-16}$ If there are both normal and mutant amplified products, then it reveals heterozygous gene variant by the existence of both normal DNA sequence and mutant DNA sequence within an individual.

In this study, evaluation of the ARMS technique for prenatal gene diagnosis of CHM with $\mathrm{CHM}$ gene point mutation was performed. This is a cheap, fast, accurate, and reliable pre- and postnatal diagnostic technique which provides the possibility to use it in the identification of various X-linked recessive gene mutations in western China and other undeveloped or developing countries.

\section{Materials and methods Clinical diagnosis and sample collection}

A Chinese proband with CHM was diagnosed previously (Figure 1A). ${ }^{17}$ DNA templates from the proband and his family of peripheral leukocytes and from the amniotic fluid of fetus at 17 weeks of pregnancy, were isolated using a previously described method. ${ }^{18,19}$ The quality of DNA was measured by a NanoDrop-2000-spectrophotometer. High quality intact genomic DNA with an optical density ratio of 260/280 1.8 and 260/230>1.5 were used for further analysis. The study was approved by the ethical committee of Southwest Medical University according to the Helsinki Declaration (1983 Revision). Pregnant women signed informed consent forms for approval of the prenatal diagnosis.
A

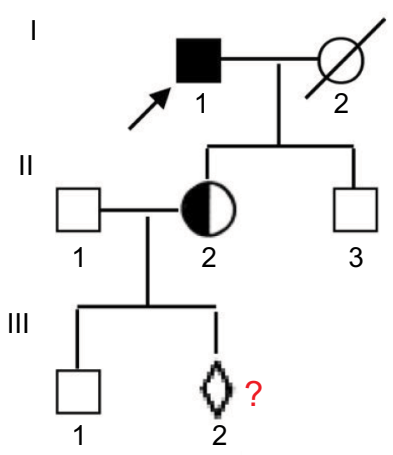

B

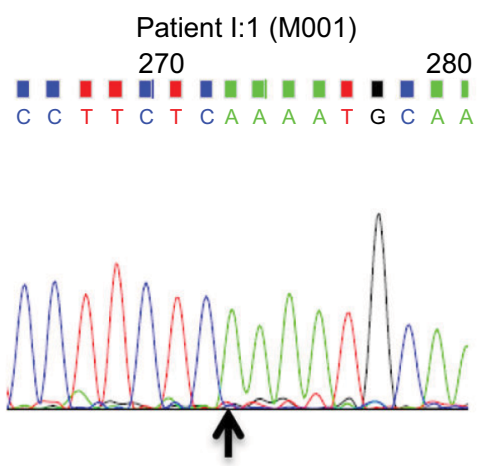

C

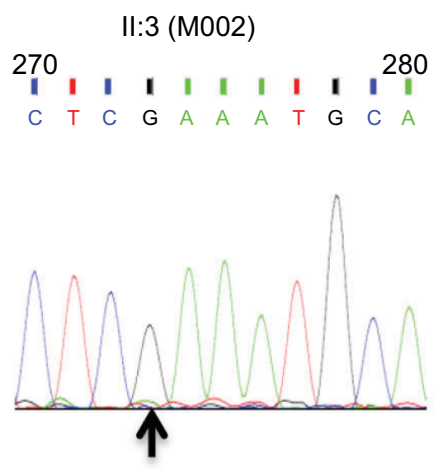

Figure I The pedigree of choroideremia and $\mathrm{CHM}$ gene mutation.

Notes: (A) Pedigree of choroideremia. (B) The proband (I:I) with CHM nonsense variant (CHM:NM_000390:c.C799T:p.R267X) is indicated by an arrow as a filled square, exhibiting a $799 \mathrm{C} \rightarrow$ T mutation at the first nucleotide position of codon 267 (R267X) in the exon 6 of the CHM gene locus (cga-tga). (C) The proband's son (II:3) harbors normal DNA sequences. The carrier (II:2) is a female of proband's daughter and is pregnant with a fetus (III:2) who underwent prenatal diagnosis to demonstrate whether the baby inherited a normal X-chromosome. All of the Sanger sequencing results in this study used reverse complementary sequencing of the gene. The black arrow shows the position of the point mutation. The primer pair of CHM-L and CHM-R was used for PCR amplification and primer CHM-R for Sanger sequencing. 


\section{Design of ARMS' primers}

We designed the $5^{\prime}$ ARMS' primers with wild and mutant types with or without one base mismatched at the $3^{\prime}$-end following the nonsense mutation (c.C799T:p.R267X) of the $C H M$ gene, respectively. ${ }^{5}$ In addition, a common primer located at the $3^{\prime}$ terminus of $C H M$ gene was designed. Table 1 presents the information of primers.

\section{ARMS-PCR amplification}

PCR was performed in a $10 \mu \mathrm{L}$ reaction volume for DNA samples M001, M002, M003, M004, M050, and M051 that comprised $1 \mu \mathrm{L}$ DNA template ( $15 \mathrm{ng}$ ), $1 \mu \mathrm{L} 3.3 \mu \mathrm{M}$ primers, $5 \mu \mathrm{L} 2 \mathrm{X}$ PCR Taq Master Mix (TianGen Biotech Co. Ltd., Beijing, People's Republic of China), and $3 \mu \mathrm{L}$ doubledistilled water. PCR cycles were performed in a programmable thermal cycler (Applied Biosystems Veriti ${ }^{\circledR}$ 96-Well Thermal Cycler, Life Technologies, Foster City, CA, USA). ${ }^{20}$ PCR regimen was as follows: initial denaturation at $95^{\circ} \mathrm{C}$ for $60 \mathrm{~s}$, followed by 33 cycles for $30 \mathrm{~s}$ at $94^{\circ} \mathrm{C}, 30 \mathrm{~s}$ at $61^{\circ} \mathrm{C}$, $40 \mathrm{~s}$ at $72^{\circ} \mathrm{C}$, and then a final extension for $5 \mathrm{~min}$ at $72^{\circ} \mathrm{C}$, and finally the PCR products were maintained at $4^{\circ} \mathrm{C}$ in the end. In case of M151, which was amniotic fluid DNA sample with a lower concentration, we increased the total cycles to 35 , and the rest of the conditions were same as the others.

\section{Agarose gel electrophoresis}

PCR products were resolved on a $1 \%$ agarose gel with $1 x$ Tris-acetate-ethylenediaminetetraacetic acid (TAE) buffer, and then subjected to electrophoresis at $120 \mathrm{~V}$ for $30 \mathrm{~min}$. The agarose gel was stained with $0.5 \mu \mathrm{g} / \mathrm{mL}$ ethidium bromide and photographed by ChemiDocXR (Bio-Rad, Hercules, CA, USA). ${ }^{19,20}$ DNA Marker (DL2000) for electrophoresis was purchased from TaKaRa Biotechnology Co. Ltd., (Dalian, People's Republic of China).

\section{Mutation validation}

To validate the ARMS-PCR results, the variant was sequenced by Sanger method on an ABI 3500 DX Genetic Analyzer (ABI, Thermo Fisher Scientific, Waltham, MA, USA) with primer CHM-R by amplification using the primer pair CHM-L and CHM-R (length of the product is $637 \mathrm{bp}$ ) (Table 2).

\section{Results}

\section{Designing of ARMS amplification primers}

In this study, we recruited a Chinese pedigree with CHM to perform the analysis using ARMS technique(Figure 1A). The results by Sanger sequencing with a variant (c.C799T:p. $\mathrm{R} 267 \mathrm{X}$ ) in the exon 6 of the CHM gene (GenBank accession number: NM_000390) on the X chromosome in the proband (I:1) and his son (II:3) with normal phenotype are shown in Figure 1B and C, respectively. As seen in Figure 1, I:1 (M001) is the proband of pedigree (indicated by an arrow) with $C H M$ gene nonsense mutation from $\mathrm{C} \rightarrow \mathrm{T}$ (Figure 1B), whereas proband's son (II:3) was normal both in phenotype and genotype with no mutation (Figure 1C). His daughter was a carrier with an unborn fetus which was tested for inheritance of the mutant gene from the mother.

In order to establish and optimize the ARMS condition, two wild-type primer sets were designed: wild-type primer (CHM-WT) and point mutation wild-type primer (CHM-WT2) which matched with wild-type sequence of CHM, sharing the $3^{\prime}$ common primer (CHM-C3) as primer pairs $(\mathrm{WT}+\mathrm{C})$ and $(\mathrm{WT} 2+\mathrm{C})$; two mutant primer sets were designed: mutant-type primer (CHM-MT) and point mutation for mutant-type primer (CHM-MT2) which matched with mutant-type sequence of CHM c.C799T, sharing the $3^{\prime}$ common primer $(\mathrm{CHM}-\mathrm{C} 3)$ as primer pairs $(\mathrm{MT}+\mathrm{C})$ and $(\mathrm{MT} 2+\mathrm{C})$ (Table 1). The length of the PCR product was 490

Table I Amplification refractory mutation system and their combination

\begin{tabular}{llllll}
\hline $\begin{array}{l}\text { Primer } \\
\text { combination }\end{array}$ & $\begin{array}{l}\mathbf{5}^{\prime} \text {-specific } \\
\text { primer }\end{array}$ & Sequence (5'-3') & $\begin{array}{l}\mathbf{3}^{\prime} \text {-common } \\
\text { primer }\end{array}$ & Sequence (5'-3') & Size (bp) \\
\hline WT+C & CHM-WT & ATTACCAGGATTCTTGCATTTC & CHM-C3 & GCTCTGCTCTTCTGGAAGCTG & 490 \\
MT $+C$ & CHM-MT & ATTACCAGGATTCTTGCATTTI & CHM-C3 & GCTCTGCTCTTCTGGAAGCTG & 490 \\
WT2+C & CHM-WT2 & ATTACCAGGATTCTTGCATCTC & CHM-C3 & GCTCTGCTCTTCTGGAAGCTG & 490 \\
MT2 $+C$ & CHM-MT2 & ATTACCAGGATTCTTGCATCTI & CHM-C3 & GCTCTGCTCTTCTGGAAGCTG & 490 \\
\hline
\end{tabular}

Abbreviations: WT, wild-type primer; $\mathrm{MT}$, mutant primer; $\mathrm{C}$, common primer; $\mathrm{CHM}$, choroideremia.

Table 2 Sequences of PCR primers and PCR product size (bp)

\begin{tabular}{lllllll}
\hline Name & $\mathbf{5}^{\prime}$-primer & Sequence $\left(\mathbf{5}^{\prime}-\mathbf{3}^{\prime}\right)$ & $\mathbf{3}^{\prime}$-primer & Sequence $\left(\mathbf{5}^{\prime} \mathbf{3}^{\prime}\right)$ & Size & Tm $\left({ }^{\circ} \mathbf{C}\right)$ \\
\hline CHM & CHM-L & TGGGAGAAAAGGATTTGTGTG & CHM-R & ATGGATCAGGTTTTGCTGCT & 637 & 60 \\
\hline
\end{tabular}

Note: $C H M-L$ was used for Sanger sequencing.

Abbreviations: L, left; R, right; bp, base pair; CHM, choroideremia. 
bp. The artificial mismatch at the 3 rd position of $3^{\prime}$-end in both CHM-WT2 and CHM-MT2 were designed to enhance PCR amplification.

The combination of primer pair $(\mathrm{MT}+\mathrm{C})$, which matched with the mutant $C H M$ gene c.C799T, was used to detect the mutant, and the combination of primer pair (MT2+C) with one base mismatch was used to enhance the specificity for detecting the mutant CHM gene c.C799T.

\section{Optimization of ARMS amplification}

PCR amplification was performed to optimize ARMS condition by the aforementioned four primer pairs using DNA templates M001 (I:1) and M002 (II:3). As seen in Figure 2, all four primer pairs amplified specific bands with right size, except the primer pair $(\mathrm{WT}+\mathrm{C})$, with no mismatch in wild-type sequence and amplified nonspecific band in M001 (Figure 2, lane labeled "WT+C"). As we know, M001 is a male patient with T mutant allelic CHM gene (Figure 1B). The primer pair $(\mathrm{WT} 2+\mathrm{C})$, with one base mismatch in wild-type sequence, amplified specific band only in normal person (M002) but not in patient (M001) (Figure 2, lane labeled "WT2+C"). Both primer pairs (MT+C and MT2+C) amplified specific bands in the proband patient M001 with the mutant allele T (Figure 2, lanes labeled "MT+C" and "MT2+C") but amplified nonspecific band in M002 (Figure 2, lanes labeled "MT+C" and "MT2+C"), indicating that there is no difference between these two primer

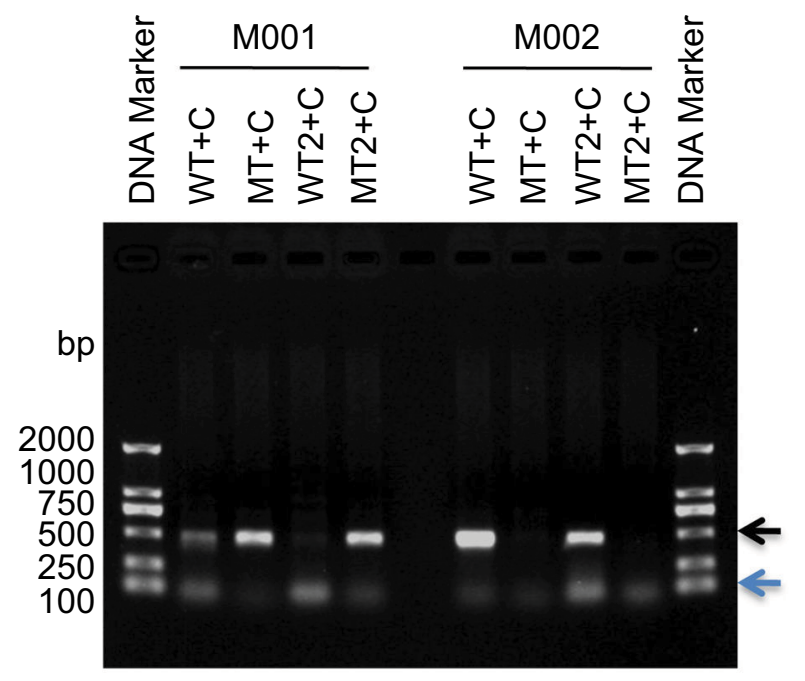

Figure 2 Optimization of ARMS condition.

Notes: ARMS amplifications specific for the mutation (c.C799T) were optimized by indicated different primer pairs. The genomic samples of mutant hemizygous proband MOOI (I:I) and his normal son (Il:3) were used as DNA templates. The black and blue arrows show the position of the point mutation and nonspecific primer-dimer, respectively. I: I=M00I; II:3=M002.

Abbreviations: ARMS, amplification refractory mutation system; WT, wild-type primer; MT, mutant primer; C, common primer. pairs. Thus, we optimized the ARMS condition by selecting primer pair (WT2+C) of wild-type and primer pair $(\mathrm{MT}+\mathrm{C})$ of mutant-type.

\section{Evaluation of ARMS for gene diagnosis of CHM}

To complete the diagnosis of CHM, we used the primer pairs $\mathrm{WT} 2+\mathrm{C}$ and $\mathrm{MT}+\mathrm{C}$ to amplify different DNA samples from all the family members and a normal male control (sample M051). As shown in Figure 3A, the patient's DNA (M001) amplified the mutant allele but did not amplify the wild-type allele, which is consistent with our previous results (shown in Figure 2); however, carrier daughter's DNA (M003) amplified both alleles. Sanger sequencing demonstrated that the daughter is heterozygous with one allele point mutation of CHM c.C799T (Figure 3B). All the normal individuals' DNA (M002, M004, M050, including control M051) amplified only wild-type allele. Validation of the putative mutation was performed by Sanger sequencing (Figure 3B and data not shown). The carrier M003 had a heterozygous variant from $\mathrm{C} \rightarrow \mathrm{T}$ and the mutant gene was same as her father, indicating that the mutant $C H M$ gene is inherited from her father (Figure 1B and 3B).

\section{Evaluation of ARMS for quick and accurate prenatal gene diagnosis of CHM} In this pedigree, M151 was the fetal baby of the carrier M003, and we amplified genetic material of M151 from amniotic fluid DNA using the same primers (WT2+C and MT $+\mathrm{C}$ ) as for other family members used above. To evaluate ARMS for quick and accurate prenatal gene diagnosis of CHM, ARMSPCR was performed and results are shown in Figure 4A, which indicates that the fetus (M151) amplified both mutant as well as the wild-type allele, similar to his/her mother (M003). Sanger sequencing result of M151 further revealed a heterozygous mutation from $\mathrm{C} \rightarrow \mathrm{T}$ (Figure 4B), confirming our ARMS results. PCR for $S R Y$ gene demonstrated that there was no Y chromosome, indicating that M151 was a female fetus which was demonstrated after birth. Thus, ARMS was performed very quickly, and its results were found to be very reliable and accurate for $C H M$ gene prenatal diagnosis.

Therefore, the female fetus M151 has the same genotype as her mother by inheriting the pathogenic mutant gene from her grandfather passing on to her mother and then to herself. With genetic counseling, the mother decided to give birth to the baby, showing no symptoms of CHM with more than 3 years' of follow-up information. 
A

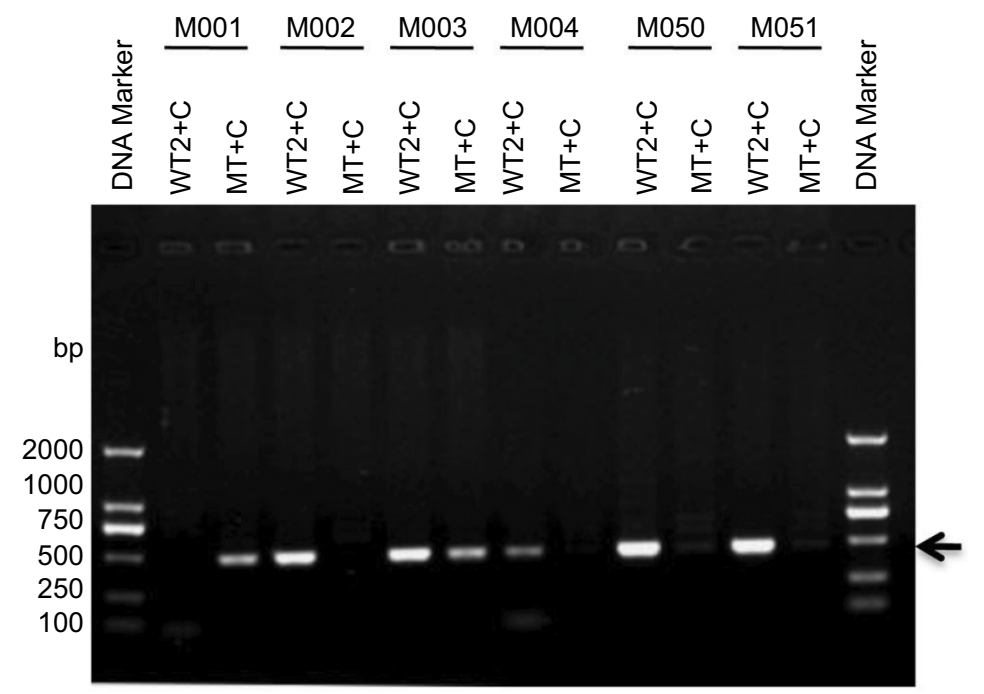

B
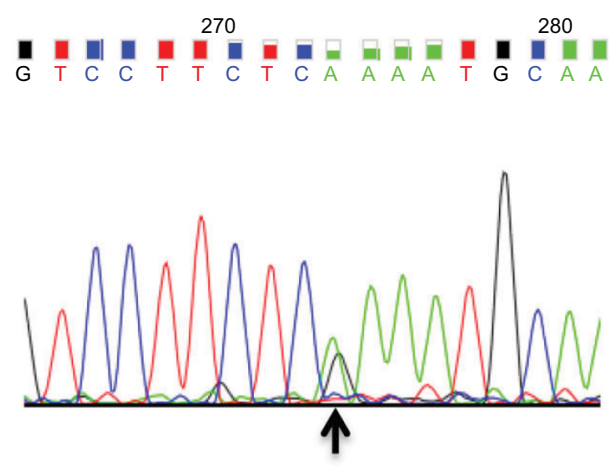

Figure 3 ARMS amplification by primers CHM-WT2 (WT2) and CHM-Common, and primers CHM-MT and CHM-Common.

Notes: (A) ARMS amplification for different DNA samples. The black arrow shows the specific PCR products. (B) Verification of M003 by Sanger sequencing. The black arrow shows the position of the point mutation. I: I=M00I; II:3=M002; II:2=M003; III:I=M004; II:I=M050; M05I=Normal male control.

Abbreviations: ARMS, amplification refractory mutation system; CHM, choroideremia; WT, wild-type primer; MT, mutant primer; C, common primer.

A

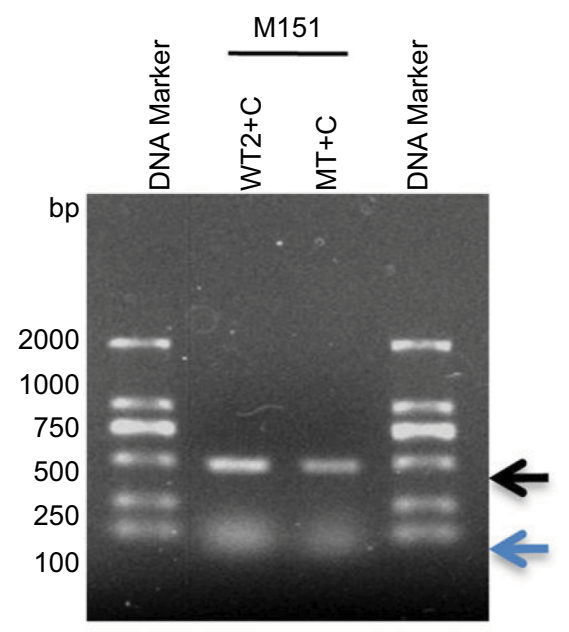

B III:2 (M151)
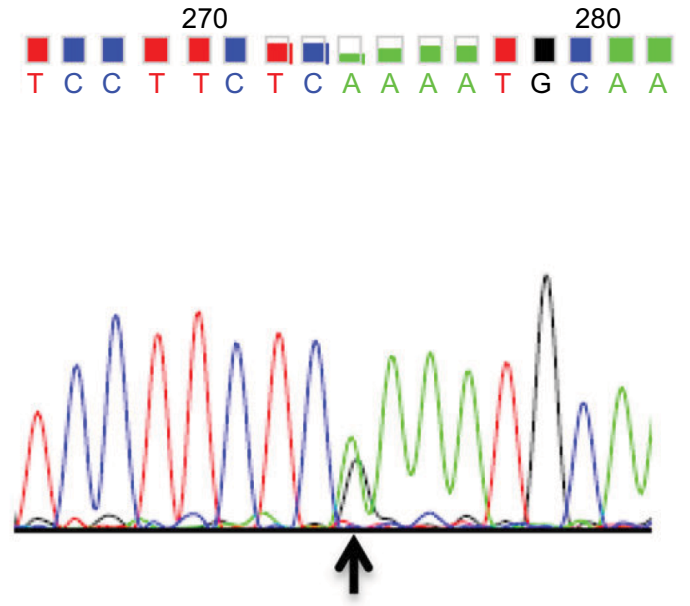

Figure 4 ARMS amplification from fetal amniotic fluid DNA (III:2) by primers CHM-WT2 (WT2) and Common primer CHM-C3, and primers CHM-MT and CHM-Common. Notes: (A) ARMS amplification. The black and blue arrows show the position of the point mutation of the specific PCR products and nonspecific primer-dimer, respectively. (B) Verification of MI5I (III:2) by Sanger sequencing. The black arrow shows the position of point mutation. A heterozygous mutation with the (c.C799T:p.R267X) variant was identified (A) and confirmed (B). III:2=MI5I.

Abbreviations: ARMS, amplification refractory mutation system; CHM, choroideremia; WT, wild-type primer; MT, mutant primer; C, common primer.

\section{Discussion}

As a rare $\mathrm{X}$-linked recessive inherited disorder, CHM causes chorioretinal dystrophy with typical clinical features such as progressive degeneration of the choriocapillaris, RPE, and photoreceptors. ${ }^{2,6,21}$ Different kinds of variants/mutations have been identified in $C H M$ gene, ${ }^{6,8,9}$ and also some methods have been applied to detect these mutations. ${ }^{4,21}$ Gene therapy and retinal transplantation of CHM are considered possible treatments and are in clinical trials from last few years. Thirteen different human retinal gene therapy clinical trials have been performed. The majority of these studies used adenoassociated virus type 2 (AAV2) as the gene transfer vector. ${ }^{22,23}$ To date, the safety assessment of gene therapy mediated by AAV2 in CHM patients has been finished already. ${ }^{21}$ Another clinical study demonstrated significant progress and suggests that AAV8 might be a candidate vector for $C H M$ gene 
therapy. ${ }^{24}$ Therefore, individuals affected with CHM might be cured in the near future. However, gene therapy has still a long way to go in order to become a reality. Thus, prenatal diagnosis is the current used strategy that can prevent genetic disorders.

Mutations in $C H M$ gene are known to lead CHM. ${ }^{25,26} \mathrm{Li}$ et al reported that at least 147 kinds of mutations have been identified in patients with CHM, containing 39 nonsense mutations, 2 missense mutations, 25 splicing mutations, 32 small deletions, 5 small indels, 9 small insertions, 1 gross insertions/duplication, 31 gross deletions, and 3 complex rearrangements, based on $H G M D \AA$ Professional 2012.4. All the aforementioned kinds of mutations are loss of function mutations except two types. ${ }^{8,27}$ In another study, they found 6 mutations including 1 nonsense, 2 splicings, and 3 small deletions, ${ }^{28}$ and all of them are loss of function mutations. Cai et al added two novel mutations to expand the spectrum of CHM-related mutations in two unrelated Chinese families. ${ }^{29}$ Guo et al discovered one frameshift mutation in a Chinese family. ${ }^{30}$ In our studies, the mutation was identified by NGS and noninvasive prenatal testing (NIPT) of SRY gene. ${ }^{17}$

ARMS is a very technique that can detect known mutations involving single base changes or small deletions, by using sequence-specific PCR primers. In this study, we successfully applied this technique to evaluate the value of prenatal gene diagnosis for CHM. To the best of our knowledge, this is the first report on gene and prenatal gene diagnosis of CHM by ARMS. Figure 5 shows the diagram of ARMS strategy for $C H M$ gene diagnosis by introducing one base mismatch $\mathrm{T} \rightarrow \mathrm{C}$ at $3 \mathrm{rd}$ position from $3^{\prime}$ end of both normal and mutant primers in our study.

Previously, Fu et al and Moghadam et al successfully used ARMS to diagnose $\beta$-thalassemia prenatally in China and Iran, respectively; ${ }^{18,31}$ Chiu et al applied this method to quantify given mutant mtDNA heteroplasmies, ${ }^{32}$ whereas Newton et al used ARMS for antenatal diagnosis of cystic fibrosis. ${ }^{33}$ Recently, Yang et al performed rapid gene diagnosis for retinitis pigmentosa, ${ }^{34}$ and Singh et al performed prenatal gene diagnosis for sickle cell disease by ARMS. ${ }^{35}$ More recently, Aquino et al performed ARMS-PCR to detect the most common mutations of the CFTR gene in Peruvian patients with cystic fibrosis. ${ }^{36}$ Shah et al combined ARMS and DNA sequencing to diagnose $\beta$-thalassemia in East-Western Indian population for better management. ${ }^{37}$ From the above instances, it is noteworthy that ARMS can be used in the pre- and postnatal diagnosis of various genetic diseases. ${ }^{38}$ Although there are many sophisticated tools applied to clinical diagnosis, it is very expensive for people in western
A

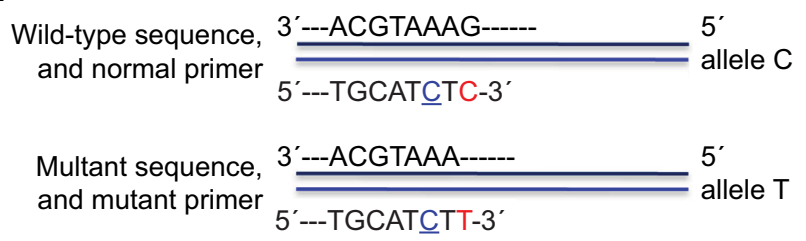

B

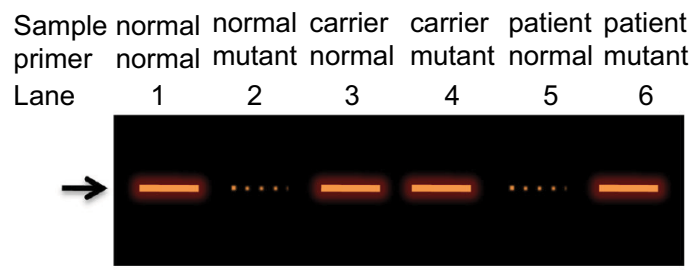

Figure 5 A diagram for ARMS strategy to diagnose $C H M$ gene.

Notes: (A) The normal and mutant target DNA sequences of $\mathrm{CHM}$ gene (black and blue solid lines) and the normal and mutant primers are depicted. One base mismatch $\mathrm{T} \rightarrow C$ was introduced artificially at $3 \mathrm{rd}$ position (blue with underline) from $3^{\prime}$ end of both normal and mutant primers in our study. (B) Representative PCR products obtained by ARMS are interpreted from an agarose gel. Lane I presents the results from a normal individual using the ARMS primer with the normal or wildtype sequence, resulting in the target DNA product (solid line). Lane 2 also presents the results from a normal individual, but the ARMS primer now corresponds to the mutant sequence; thus, PCR amplification does not occur (dotted line). Lanes 3 and 4 reveal the results with a heterozygous individual, carrier. In lane 3 , the ARMS primer corresponds to wild-type sequence with PCR amplification of target DNA (from one allele C) (solid line) and in lane 4, the ARMS primer corresponds to mutant sequence with PCR amplification of target DNA (from the other allele T) (solid line). Lanes 5 and 6 reveal the results from a male patient with the mutation on an allele $\mathrm{T}$ in $\mathrm{X}$ chromosome gene CHM (hemizygote). Lane 5 represents the results with an ARMS primer that corresponds to wild-type sequence with no PCR amplification (dotted line); lane 6 uses an ARMS primer that corresponds to the mutant sequence, with amplification of target DNA (solid line). The black arrow points to the specific PCR products.

Abbreviations: ARMS, amplification refractory mutation system; CHM, choroideremia.

China and some other undeveloped countries. As for known mutations in genes, ARMS provides an optional way to solve this problem with its additional characteristics such as timeefficient, unbiased, sensitive, accurate, rapid, and reliable. In our study, we successfully developed the ARMS technique by using wild-type or mutant-type primers with matched or one-base mismatched to examine the known mutation in the CHM gene. Furthermore, we tried to obtain samples to test how early ARMS method can be applied in clinical settings, and currently, we are isolating cff DNAs from different stages to test whether ARMS work.

Taken together, ARMS could be useful for quick and accurate prenatal gene diagnosis of CHM for CHM gene as well as other genetic diseases in undeveloped and developing countries which are in shortage of medical resources and supplies.

\section{Acknowledgments}

This work was supported by the Research Foundation of the Education Department of Sichuan Province 
(17ZA0427,17ZB0467), the Research Foundation of the Science and Technology Department of Luzhou City (2013LZLY-J10, 2016-S-65(9/9), 2015-S-42(3/4)), and in part by the National Natural Science Foundation of China (Nos. 30371493, 31701087, 81672887, and 81172049).

\section{Disclosure}

The authors report no conflicts of interest in this work.

\section{References}

1. Roberts MF, Fishman GA, Roberts DK, et al. Retrospective, longitudinal, and cross sectional study of visual acuity impairment in choroideraemia. Br J Ophthalmol. 2002;86(6):658-662.

2. Coussa RG, Traboulsi EI. Choroideremia: a review of general findings and pathogenesis. Ophthalmic Genet. 2012;33(2):57-65.

3. Rudolph G, Preising M, Kalpadakis P, Haritoglou C, Lang GE, Lorenz B. Phenotypic variability in three carriers from a family with choroideremia and a frameshift mutation 1388delCCinsG in the REP-1 gene. Ophthalmic Genet. 2003;24(4):203-214.

4. Dimopoulos IS, Radziwon A, St Laurent CD, MacDonald IM. Choroideremia. Curr Opin Ophthalmol. 2017;28(5):410-415.

5. Cremers FP, van de Pol DJ, van Kerkhoff LP, Wieringa B, Ropers H-H. Cloning of a gene that is rearranged in patients with choroideremia. Nature. 1990;347(6294):674-677.

6. Van Bokhoven H, van den Hurk JA, Bogerd L, et al. Cloning and characterization of the human choroideremia gene. Hum Mol Genet 1994;3(7):1041-1046.

7. Ran X, Cai WJ, Huang XF, et al. 'RetinoGenetics': a comprehensive mutation database for genes related to inherited retinal degeneration. Database (Oxford). 2014;2014:bau047.

8. Sergeev YV, Smaoui N, Sui R, et al. The functional effect of pathogenic mutations in Rab escort protein 1. Mutat Res. 2009;665(1):44-50.

9. Itabashi T, Wada Y, Kawamura M, Sato H, Tamai M. Clinical features of Japanese families with a $402 \mathrm{delT}$ or a $555-556 \mathrm{del}$ AG mutation in choroideremia gene. Retina. 2004;24(6):940-945.

10. Seabra MC, Brown MS, Slaughter CA, Südhof TC, Goldstein JL. Purification of component A of Rab geranylgeranyl transferase: possible identity with the choroideremia gene product. Cell. 1992;70(6):1049-1057.

11. Pereiraleal JB, Hume AN, Seabra MC. Prenylation of Rab GTPases: molecular mechanisms and involvement in genetic disease. FEBS Lett. 2001;498(2-3):197-200.

12. Goody RS, Rak A, Alexandrov K. The structural and mechanistic basis for recycling of Rab proteins between membrane compartments. Cell Mol Life Sci. 2005;62(15):1657-1670.

13. Fu Q, Wang F, Wang H, et al. Next-generation sequencing-based molecular diagnosis of a Chinese patient cohort with autosomal recessive retinitis pigmentosa. Invest Ophthalmol Vis Sci. 2013;54(6):4158-4166.

14. Newton CR, Graham A, Heptinstall LE, et al. Analysis of any point mutation in DNA. The amplification refractory mutation system (ARMS). Nucleic Acids Res. 1989;17(7):2503-2516.

15. Hassan S, Ahmad R, Zakaria Z, Zulkafli Z, Wan ZA. Detection of $\beta$-globin gene mutations among $\beta$-thalassaemia carriers and patients in Malaysia: application of multiplex amplification refractory mutation system-polymerase chain reaction. Malays J Med Sci. 2013;20(1):13-20.

16. Hanafi S, Hassan R, Bahar R, et al. Multiplex amplification refractory mutation system (MARMS) for the detection of $\beta$-globin gene mutations among the transfusion-dependent $\beta$-thalassemia Malay patients in Kelantan, Northeast of Peninsular Malaysia. Am J Blood Res. 2014;4(1):33-40.

17. Zhu L, Cheng J, Zhou B, et al. Diagnosis for choroideremia in a large Chinese pedigree by next-generation sequencing (NGS) and non-invasive prenatal testing (NIPT). Mol Med Rep. 2017;15(3):1157-1164.
18. Fu JJ, Li LY, Li XR, Lu GX. Rapid prenatal gene diagnosis for $\beta$-thalassemia by amplification refractory mutation system (ARMS). Chin J Obstet Gynecol. 2000;35:359-360.

19. Fu J. Short Protocols in Medical Molecular Biology. Beijing: China Medical Science Press; 2012.

20. Fu J, Yang L, Khan MA, Mei Z. Genetic characterization and authentication of Lonicera japonica Thunb. by using improved RAPD analysis. Mol Biol Rep. 2013;40(10):5993-5999.

21. Maclaren RE, Groppe M, Barnard AR, et al. Retinal gene therapy in patients with choroideremia: initial findings from a phase $1 / 2$ clinical trial. Lancet. 2014;383(9923):1129-1137.

22. Amado D, Mingozzi F, Hui D, et al. Safety and efficacy of subretinal readministration of an AAV2 vector in large animal models: implications for studies in humans. Sci Transl Med. 2010;2(21):21ra16.

23. Bennett J, Ashtari M, Wellman J, et al. AAV2 gene therapy readministration in three adults with congenital blindness. Sci Transl Med. 2012;4(120):120ra15.

24. Black A, Vasireddy V, Chung DC, et al. Adeno-associated virus 8-mediated gene therapy for choroideremia: preclinical studies in in vitro and in vivo models. J Gene Med. 2014;16(5-6):122-130.

25. Cremers FP, Armstrong SA, Seabra MC, Brown MS, Goldstein JL. REP-2, a Rab escort protein encoded by the choroideremia-like gene. J Biol Chem. 1994;269(3):2111-2117.

26. Ying L, Xialin L, Lixia L, et al. Molecular analysis of the choroideremia gene related clinical findings in two families with choroideremia. Mol Vis. 2011;17(277):2564-2569.

27. Esposito G, De Falco F, Tinto N, et al. Comprehensive mutation analysis (20 families) of the choroideremia gene reveals a missense variant that prevents the binding of REP1 with rab geranylgeranyl transferase. Hum Mutat. 2011;32(12):1460-1469.

28. Li S, Guan L, Fang S, et al. Exome sequencing reveals CHM mutations in six families with atypical choroideremia initially diagnosed as retinitis pigmentosa. Int J Mol Med. 2014;34(2):573-577.

29. Cai XB, Huang XF, Tong Y, Lu QK, Jin ZB. Novel CHM mutations identified in Chinese families with Choroideremia. Sci Rep. 2016;6:35360.

30. Guo H, Li J, Gao F, Li J, Wu X, Liu Q. Whole-exome sequencing reveals a novel CHM gene mutation in a family with choroideremia initially diagnosed as retinitis pigmentosa. BMC Ophthalmol. 2015;15:85.

31. Moghadam M, Karimi M, Dehghani SJ, et al. Effectiveness of $\beta$-thalassemia prenatal diagnosis in Southern Iran: a cohort study. Prenat Diagn. 2015;35(12):1238-1242.

32. Chiu RW, Murphy MF, Fidler C, Zee BC, Wainscoat JS, Lo YM. Determination of RhD zygosity: comparison of a double amplification refractory mutation system approach and a multiplex real-time quantitative PCR approach. Clin Chem. 2001;47(4):667-672.

33. Newton CR, Heptinstall LE, Summers C, et al. Amplification refractory mutation system for prenatal diagnosis and carrier assessment in cystic fibrosis. Lancet. 1989;2(8678-8679):1481-1483.

34. Yang WC, Zhu L, Zhou BX, et al. Establishment and rapid detection of a heterozygous missense mutation in the CACNA1F gene by ARMS technique with double-base mismatched primers. Genet Mol Res. 2015;14(3):11480-11487.

35. Singh PJ, Shrivastava AC, Shrikhande AV. Prenatal diagnosis of sickle cell disease by the technique of PCR. Indian J Hematol Blood Transfus. 2015;31(2):233-241.

36. Aquino R, Protzel A, Rivera J, et al. [Frequency of the most common mutations of the CFTR gene in peruvian patients with cystic fibrosis using the ARMS-PCR technique]. Rev Peru Med Exp Salud Pública. 2017;34(1):62-69. Spanish.

37. Shah PS, Shah ND, Ray HSP, et al. Mutation analysis of $\beta$-thalassemia in East-Western Indian population: a recent molecular approach. Appl Clin Genet. 2017;10:27-35.

38. Imani S, Cheng J, Shasaltaneh MD, et al. Genetic identification and molecular modeling characterization reveal a novel PROM1 mutation in Stargardt4-like Macular Dystrophy. Oncotarget. In press 2017. 


\section{Publish your work in this journal}

The Application of Clinical Genetics is an international, peer-reviewed open access journal that welcomes laboratory and clinical findings in the field of human genetics. Specific topics include: Population genetics; Functional genetics; Natural history of genetic disease; Management of genetic disease; Mechanisms of genetic disease; Counselling and ethical issues; Animal models; Pharmacogenetics; Prenatal diagnosis; Dysmorphology. The manuscript management system is completely online and includes a very quick and fair peer-review system, which is all easy to use. Visit http://www.dovepress.com/testimonials.php to read real quotes from published authors.

Submit your manuscript here: https://www.dovepress.com/the-application-of-clinical-genetics-journal 